\title{
On Magnetization Reversal in Hard Magnetic Sm-Fe-N Permanent Magnets
}

\author{
P. PAWLIK, J.J. WYSŁOCKI* \\ Institute of Physics, Technical University of Częstochowa \\ al. Armii Krajowej 19, 42-200 Częstochowa, Poland \\ AND M. BARAN \\ Institute of Physics, Polish Academy of Sciences \\ al. Lotników 32/46, 02-668 Warszawa, Poland
}

(Received August 6, 2001)

\begin{abstract}
The investigations were carried out on $\mathrm{Sm}-\mathrm{Fe}-\mathrm{N}$ permanent magnets produced by reactive diffusion method. The magnets consist of hard magnetic phases: $\mathrm{Sm}_{2} \mathrm{Fe}_{17} \mathrm{~N}_{0.86}$ and $\mathrm{SmFe}_{5}$ and soft magnetic $\alpha$-Fe phase. From the comparison of experimentally determined angular dependence of coercive field with appropriate theoretical predictions and from the dependence of coercive field on the external magnetic field determined from the minor hysteresis loops, it was stated that magnetization reversal process in $\mathrm{Sm}-\mathrm{Fe}-\mathrm{N}$ magnets is controlled by the nucleation of reversed domains process.
\end{abstract}

PACS numbers: 75.50.-y, 75.60.-d

\section{Introduction}

$\mathrm{Sm}_{2} \mathrm{Fe}_{17} \mathrm{~N}_{3}$ is a recently discovered compound with excellent intrinsic magnetic properties [1]. The material possesses a very large anisotropy field estimated to be above $20 \mathrm{~T}$ at room temperature and a Curie temperature of $740 \mathrm{~K}$ [2]. The origin and formation of the magnetic properties for this group of magnets have been intensively studied [3-6], together with microstructure [7] and domain structure observations $[6,8,9]$.

*corresponding author; e-mail: wyslocki@mim.pcz.czest.pl 
Satisfactory functional properties of modernly produced magnets are related to intrinsic magnetic properties of intermetallic phases forming those magnets (like: saturation magnetization $M_{\mathrm{s}}$, anisotropy field $H_{\mathrm{a}}$, Curie temperature $T_{\mathrm{C}}$ ), optimal microstructure of the magnet (coercive field ${ }_{J} H_{c}$, maximum energy product $\left.(B H)_{\max }\right)$, as well as the chemical composition. Microstructure parameters mostly decide about magnetic reversal processes that consist in making difficult the nucleation and expansion of the reversed domains.

It should be noted that the former improvement of the functional magnetic properties has been achieved only by means of heat treatment optimization and selection of appropriate chemical composition, without deep knowledge of magnetization reversal processes in those materials.

Up to now, there is no uniform and satisfactory model describing magnetization reversal process in those materials. Therefore, in this paper the results of analysis taking into account classical models of magnetization reversal processes (Stoner-Wohlfarth [10], Jacobs-Bean [11] and Shtrikman-Treves [12] models), as well as contemporary models (Kronmüller [13-15] and Givord [16, 17] models), are presented.

In the model proposed by Kronmüller, the existence of the second magnetic phase with lower values of anisotropy constants, irregular grain surfaces, exchange coupling between grains, as well as misalignment of magnetic grains, were taken into consideration. However, in the nucleation expansion model proposed by Givord [16, 17], the existence of reversed domains in the remanent state is assumed. In this case, the critical magnetic field and thermal fluctuations of the sample determine the spontaneous expansion of these nuclei.

Therefore, the main aim of this work was to determine magnetization reversal processes in a new group of $\mathrm{Sm}-\mathrm{Fe}-\mathrm{N}$ hard magnetic materials.

\section{Material and experimental methods}

The examined samples of $\mathrm{Sm}-\mathrm{Fe}-\mathrm{N}$ magnets were prepared by reactive diffusion method that consists of pressing the mixture of $\mathrm{Sm}$ and Fe powders under the pressure of $100 \mathrm{MPa}$. Subsequently the material is annealed for $5 \mathrm{~h}$ at a temperature of $1320 \mathrm{~K}$, i.e. below the melting point of the components. As a result of solid state diffusion, a relatively isotropic crystalline structure is obtained. After the initial milling for $20 \mathrm{~min}$, the powders are nitrided for $3 \mathrm{~h}$ at a temperature of $750 \mathrm{~K}$. The final milling procedure is used in order to achieve the required grain size. In the last stage of the production process the powders are compacted with the addition of thermosetting plastic in external magnetic field, in order to obtain the aligned magnets with anisotropic properties.

In this work the samples compacted just after nitriding process, as well as after different final milling times: $2 \mathrm{~h}, 6 \mathrm{~h}, 12 \mathrm{~h}, 24 \mathrm{~h}$, and $48 \mathrm{~h}$, were examined. The related grain diameters of the powders are: $8.6 \mu \mathrm{m}, 6.6 \mu \mathrm{m}, 3.3 \mu \mathrm{m}, 2.2 \mu \mathrm{m}$, 
$1.6 \mu \mathrm{m}$, and $0.97 \mu \mathrm{m}$, respectively. During the production procedure the chemical composition of pure Sm and Fe powders was close to the stoichiometric constitution of $\mathrm{Sm}_{2} \mathrm{Fe}_{17}$ phase, i.e. 25 wt.\% of $\mathrm{Sm}$ and 75 wt.\% of $\mathrm{Fe}$.

Using an X-ray microanalyser, as well as an X-ray spectrometer with Co $K_{\alpha}$ radiation, the qualitative analysis of phase structure was performed. Furthermore, using a Mössbauer spectrometer with ${ }^{57} \mathrm{Co}$ isotope in rhodium matrix, the quantitative analysis of examined samples was carried out. Measurements of Mössbauer spectra were performed at room temperature.

It was shown that the examined samples consist of two hard magnetic phases: $\mathrm{Sm}_{2} \mathrm{Fe}_{17} \mathrm{~N}_{0.86}$ and $\mathrm{SmFe}_{5}$ and soft magnetic $\alpha$-Fe phase. Furthermore, the volume fraction of hard magnetic phase $\mathrm{Sm}_{2} \mathrm{Fe}_{17} \mathrm{~N}_{0.86}$ decreases with decreasing the grain size (from $61.88 \%$ for a $8.6 \mu \mathrm{m}$ grain diameter sample to $30.00 \%$ for a $0.97 \mu \mathrm{m}$ grain diameter sample), whereas the increase in $\mathrm{SmFe}_{5}$ phase volume fraction (from 9.00 to $59.75 \%$ ) is observed. Furthermore, a decrease in $\alpha$-Fe volume fraction from $29.12 \%$ for a grain diameter of $8.6 \mu \mathrm{m}$ to $10.25 \%$ for a grain diameter of $0.97 \mu \mathrm{m}$ was evidenced. Additional information concerning phase structure examination, performed by X-ray diffraction, as well as volume fraction analysis using Mössbauer spectroscopy, were presented in [6].

Furthermore, the investigations of magnetic properties in high external magnetic fields were carried out using a superconducting magnetometer (SQUID magnetometer).

\section{Results and discussion}

\subsection{Magnetic properties of examined magnets}

In Fig. 1 the initial magnetization curve, as well as the hysteresis loop of anisotropic Sm-Fe-N magnet with a grain diameter of $0.97 \mu \mathrm{m}$, produced by reactive diffusion method and compacted in external magnetic field with an addition of thermosetting plastic, were presented. The measurements were carried out using a SQUID magnetometer in the magnetic field up to $4 \mathrm{MA} / \mathrm{m}$. The examined samples were previously demagnetized in reversed alternating external magnetic field.

The initial magnetization curve exhibits inflection point corresponding to external magnetic field $800 \mathrm{kA} / \mathrm{m}$. For comparison in Fig. 2 the initial magnetization curve and hysteresis loop for $\mathrm{Sm}-\mathrm{Fe}-\mathrm{N}$ anisotropic magnet with a grain diameter of $8.6 \mu \mathrm{m}$, are presented. In this case, the shape of initial magnetization curve is regular and typical of the magnets with the reversal magnetization process governed by the domain nucleation [10].

Kobayashi et al. [18] have observed a similar inflection of the initial magnetization curve for single phase $\mathrm{Sm}_{2} \mathrm{Fe}_{17} \mathrm{~N}_{3}$ magnets, with a grain diameter of $3.8 \mu \mathrm{m}$. Those authors associate such shape of the initial magnetization curve with several magnetization processes, in particular: domain walls motion in low magnetic fields, 


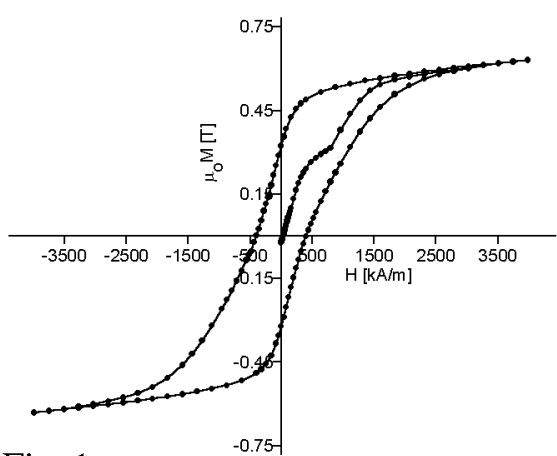

Fig. 1

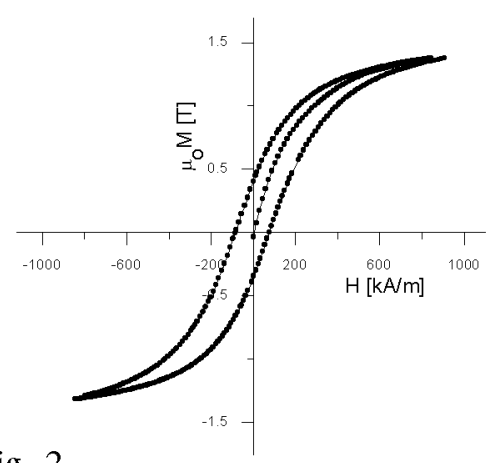

Fig. 2

Fig. 1. Initial magnetization curve and hysteresis loop for anisotropic $\mathrm{Sm}-\mathrm{Fe}-\mathrm{N}$ permanent magnet with a $0.97 \mu \mathrm{m}$ grain diameter, produced by the reactive diffusion method. Fig. 2. Initial magnetization curve and hysteresis loop for anisotropic Sm-Fe-N permanent magnet with a $8.6 \mu \mathrm{m}$ grain diameter, produced by the reactive diffusion method.

magnetization reversal of single domain particles in medium magnetic fields and rotation of magnetic moments in high magnetic fields. On the other hand, Kou et al. [19] observed a similar course of the initial magnetization curve in $\mathrm{Tb}_{2} \mathrm{Fe}_{17}$ monocrystals at a temperature of $4.2 \mathrm{~K}$. In order to explain the course of this curve, the authors assumed the coexistence of the rhombohedral and hexagonal crystalline structure in the elementary cell of the $\mathrm{Tb}_{2} \mathrm{Fe}_{17}$ compound.

However, in this work the inflection on the initial magnetization curve (Fig. 1) was associated with changes of hard magnetic phase contents - particularly with a decrease in $\mathrm{Sm}_{2} \mathrm{Fe}_{17} \mathrm{~N}_{0.86}$ phase content with decreasing the grain size (from $61.88 \%$ for a $8.6 \mu \mathrm{m}$ grain diameter to $30.00 \%$ for a $0.97 \mu \mathrm{m}$ grain diameter), an increase in $\mathrm{SmFe}_{5}$ phase content (from $9.00 \%$ to $59.75 \%$ ) and a decrease in $\alpha$-Fe soft magnetic phase content (from $29.12 \%$ to $10.25 \%$ ). Furthermore, the characteristic contraction of the hysteresis loop for the magnets with a $0.97 \mu \mathrm{m}$ grain diameter was observed, which is correlated with an earlier magnetization reversal of the phase with the lowest coercive field. For those magnets, the saturation magnetization $\mu_{0} M_{\mathrm{s}}=0.696 \mathrm{~T}$ was determined using extrapolation to the infinite external field $H \rightarrow \infty$ the dependence of magnetization $M$ on the second power of reciprocal external magnetic field $1 / H^{2}$.

On the basis of the obtained results and in particular from the comparison of initial magnetization curve courses (Figs. 1, 2) with the theoretical predictions [20], we can state that the magnetization reversal processes in the studied magnet consist in the nucleation of new reversed domains. For a further confirmation of this conclusion, the dependence of coercive field on the angle between easy direction of magnetization and external magnetic field, as well as the coercivity ${ }_{J} H_{c}$ changes determined from the minor hysteresis loops for different maximum external fields, were measured. 


\subsection{Coercive field ${ }_{J} H_{\mathrm{c}}$ dependence on the angle $\Theta$ between external magnetic field and easy direction of magnetization in $S m-F e-N$ permanent magnets}

In modern hard magnetic materials, the dominating magnetization reversal process is pinning of domain walls or nucleation of reversed domains. Changes of the coercive field as a function of angle $\Theta$ between magnetic field $H$ direction and easy axis of magnetization are often used as a test of magnetization reversal process, as was done e.g. for $\mathrm{Nd}-\mathrm{Fe}-\mathrm{B}$ [21] and $\mathrm{Fe}-\mathrm{Al}-\mathrm{C}[22]$ magnets.

For the magnets with magnetization reversal process governed by nucleation of reversal domains, the angular dependence of coercive field ${ }_{J} H_{c}(\Theta)$ should correspond to the theory of coherent rotation of magnetization vector [10]. However, for magnets controlled by the domain wall pinning mechanism, coercivity ${ }_{J} H_{c}(\Theta)$ changes should correspond to Kondorsky's relation [23] ${ }_{J} H_{c}(\Theta)={ }_{J} H_{c}(0) / \cos \Theta$ for $0 \leq \Theta \leq \pi / 2$.

In Fig. 3 the experimentally determined angular dependence of normalized coercivity ${ }_{J} H_{c}(\Theta) /{ }_{J} H_{c}(0)$ for $\mathrm{Sm}-\mathrm{Fe}-\mathrm{N}$ magnets with a grain diameter of $0.97 \mu \mathrm{m}$, is presented (denoted as curve a). For comparison in this figure the theoretical angular dependence of coercive field related to the domain wall pinning mechanism Kondorsky's relation [23] (curve d) and this relation modified by Suponev et al. [24] by considering the domain wall motion and coherent rotations of magnetization vector according to the formula (curve e):

$$
H_{\mathrm{c}}(\Theta)=H_{\mathrm{c}}(0) \frac{\cos \Theta\left(D_{\mathrm{a}}+D_{x}\right)}{D_{z} \sin ^{2} \Theta+\left(D_{\mathrm{a}}+D_{x}\right) \cos ^{2} \Theta},
$$

are plotted, where $D_{z}$ and $D_{x}\left(=D_{y}\right)$ - demagnetization coefficients for the sample in the shape of ellipsoid of revolution; $D_{\mathrm{a}}=H_{\mathrm{a}} / M_{\mathrm{s}}$ - the coefficient related to magnetocrystalline anisotropy. Furthermore, the angular dependence of coercive field was calculated according to the Stoner-Wohlfarth model [10] (curve c), and the model of domain nucleation with reversed magnetization vector [25] (curve $b$ ).

According to the Stoner-Wohlfarth theory [10], the normalized coercivity decreases from 1 to $1 / 2$, and then again increases to the initial value (curve $c$ in Fig. 3). As a result from Fig. 3, the experimental angular dependence of coercive field up to $70^{\circ}$ well corresponds to the nucleation model. Particularly, with decreasing the $\Theta$ angle, the coercivity initially decreases to the minimum value (equal to the reduced coercive field ${ }_{J} H_{c}(\Theta) /{ }_{J} H_{c}(0)=0.73$ ), corresponding to $\Theta=45^{\circ}$. Subsequently, the coercive field increases with increasing the $\Theta$ angle up to the maximum value $\left({ }_{J} H_{c}(\Theta) /{ }_{J} H_{c}(0)=0.95\right)$ for $\Theta=70^{\circ}$, and then decreases to the minimum value $\left({ }_{J} H_{c}(\Theta) /{ }_{J} H_{c}(0)=0.48\right)$ for $\Theta=90^{\circ}$.

The results obtained in this work cannot be explained on the basis of Kondorsky theory [23], where the domain wall pinning is responsible for demagnetization processes, as well as the modified Kondorsky model proposed by Suponev et al. [24], where the domain wall motion (more probable for small angle $\Theta$ ) and 


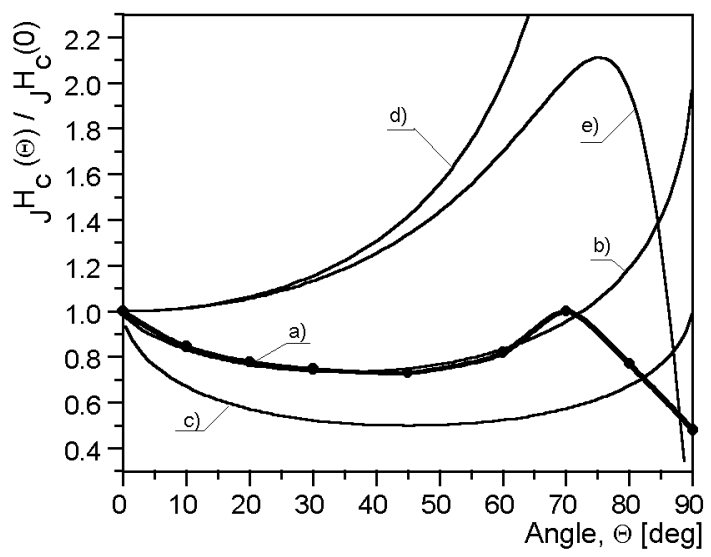

Fig. 3. The angular dependence of reduced coercive field ${ }_{J} H_{c}(\Theta) /{ }_{J} H_{c}(\Theta=0)$ for $\mathrm{Sm}-\mathrm{Fe}-\mathrm{N}$ magnet with a grain diameter of $0.97 \mu \mathrm{m}$ : a) experimental curve, b) the curve corresponding to the nucleation reversal process for $2 K_{2} / K_{1} \approx 1$ [25], c) the curve corresponding to the model of coherent rotation of magnetization vector [10], d) the curve corresponding to Kondorsky relation $1 / \cos \Theta$ [23], e) according to the modified Kondorsky relation [24].

coherent rotation of magnetization vector (characteristic for higher values of $\Theta$ angle) are considered.

On the other hand, the comparison of the curves a) and b) presented in Fig. 3, reveals that for a range of $\Theta$ angle from 0 to $70^{\circ}$, good agreement between the experimental data and predictions of the nucleation model proposed by Kronmüller and Schrefl [25] is obtained. According to this model, the changes of coercivity on $\Theta$ angle are described by the following relation:

$$
\begin{aligned}
& H_{c}(\Theta)=\frac{2 K_{1}}{\mu_{0} M_{\mathrm{s}}} \frac{1}{\left(\cos ^{2 / 3} \Theta+\sin ^{2 / 3} \Theta\right)^{3 / 2}}\left[1+\frac{2 K_{2}}{K_{1}} \frac{(\tan \Theta)^{2 / 3}}{1+(\tan \Theta)^{2 / 3}}\right] \\
& -\left(D_{z}-D_{x}\right) M_{\mathrm{s}},
\end{aligned}
$$

where $D_{z}$ and $D_{x}$ are demagnetization coefficients.

However, for a range of $\Theta$ from $70^{\circ}$ to $90^{\circ}$, the differences between experiment and theoretical predictions are evidenced. According to Suponev et al. [24] in order to explain the differences in the equation describing the angular dependence of coercivity, the domain wall motion and coherent rotation of magnetization vector should be considered. Furthermore, to explain the course of coercive field dependence on the angle $\Theta$, the existence of different magnetic phases in the examined magnets should be considered, in particular soft magnetic $\alpha$-Fe phase, for which the domain wall motion is responsible for the magnetization reversal process.

The comparison of the coercive field dependence on the angle $\Theta$ with the theoretical predictions for $\mathrm{Sm}-\mathrm{Fe}-\mathrm{N}$ magnets allows assuming that for the range 
of $\Theta$ angle between 0 and $70^{\circ}$, the magnetization reversal process is correlated to the nucleation mechanism of reversed domains. For higher values of $\Theta$ angle, the magnetization reversal process is more complex than the one predicted by the theoretical models.

3.3. Coercive field ${ }_{J} H_{\mathrm{c}}$ changes on the external magnetic field, determined from the minor hysteresis loops

Dependence of coercive field ${ }_{J} H_{\mathrm{c}}$ on the external magnetic field $H$, determined from the minor hysteresis loops, is another examination method that allows us to determine the magnetization reversal process. For the pinning controlled coercivity mechanism of the magnets, the domain walls cannot easily move through the sample and the permeability for such magnets is small until the external field gain the coercive field value. Furthermore, the drastic change of coercivity in external magnetic field equal to the coercive field, is observed as it was shown, e.g. for $\mathrm{Fe}-\mathrm{Al}-\mathrm{C}$ magnets [22]. However, for the magnets in which magnetization reversal process is correlated with nucleation of reversed domains, the existing domain walls can move easily and the initial permeability has a high value. In this case, almost linear increase in coercive field with increasing external magnetic field up to the maximum value is observed [9].

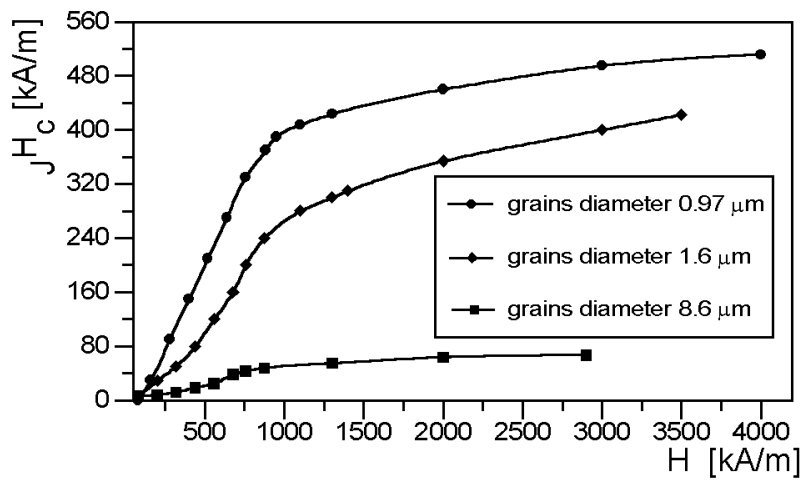

Fig. 4. The coercive field ${ }_{J} H_{c}$ dependence on the maximum external magnetic field $H$, determined from minor hysteresis loops for $\mathrm{Sm}-\mathrm{Fe}-\mathrm{N}$ magnets with different grain sizes.

That relation is presented in Fig. 4, for Sm-Fe-N magnets with grain diameters of $8.6 \mu \mathrm{m}, 1.6 \mu \mathrm{m}$, and $0.97 \mu \mathrm{m}$. The linear increase in coercive field ${ }_{J} H_{\mathrm{c}}$ with increasing external magnetic field is observed for low values of the magnetic field. It indicates that the coercive field in $\mathrm{Sm}-\mathrm{Fe}-\mathrm{N}$ magnets is associated with the mechanism of the reversed domain nucleation. 


\section{Conclusions}

The comparison of experimentally determined angular dependence of coercive field with theoretical relations, proved that for $\Theta$ angles between 0 and $70^{\circ}$ the magnetization reversal process is correlated with the nucleation of reversed domains. Another confirmation of this conclusion was provided by the course of the ${ }_{J} H_{c}(H)$ curves determined from the minor hysteresis loops for different values of the external field. The shape of those curves is typical of the magnets with the magnetization reversal process controlled by the nucleation of reversed domains.

\section{Acknowledgment}

This work was supported bythe State Committee for Scientific Research under grant No. 2 P03B 01915.

\section{References}

[1] J.M.D. Coey, H. Sun, J. Magn. Magn. Mater. 87, L251 (1990).

[2] N.M. Demsey, P.A.P. Wendhausen, B. Gebel, K.-H. Müller, J.M.D. Coey, J. Magn. Magn. Mater. 157/158, 99 (1996).

[3] K. Kobayashi, in: Proc. 13th Int. Workshop on RE Magnets and their Applications, Birmingham, September 1994, Eds. C.A.F. Manwaring, D.G.R. Jones, A.J. Williams, I.R. Harris, p. 717.

[4] S. Brennan, X.-L. Rao, R. Skomski, N. Dempsey, J.M.D. Coey, J. Magn. Magn. Mater. 157/158, 510 (1996).

[5] P. Pawlik, J.J. Wysłocki, W. Kaszuwara, M. Leonowicz, Mater. Sci. Forum 373-376, 309 (2001).

[6] P. Pawlik, J.J. Wysłocki, J. Olszewski, K. Pawlik, submitted to Acta Phys. Pol. A.

[7] K. O'Donnell, M. Aindow, I.R. Harris, R. Skomski, J.M.D. Coey, J. Magn. Magn. Mater. 157/158, 79 (1996).

[8] P. Pawlik, J.J. Wysłocki, W. Kaszuwara, M. Leonowicz, in: Proc. 15th Int. Workshop on RE Magnets and their Applications, Dresden, August/ September 1998, Eds. L. Schultz, K.-H. Müller, p. 165.

[9] Jifan Hu, T. Dragon, M.-L. Sartorelli, H. Kronmüller, Phys. Status Solidi A 136, 207 (1993).

[10] E.C. Stoner, E.P. Wohlfarth, Philos. Trans. R. Soc. Lond. A 240, 599 (1948).

[11] I.S. Jacobs, C.P. Bean, Phys. Rev. 100, 1060 (1955).

[12] S. Shtrikman, D. Treves, J. Phys. Radium 20, 286 (1959).

[13] H. Kronmüller, K.-D. Durst, M. Sagawa, J. Magn. Magn. Mater. 74, 291 (1988).

[14] H. Kronmüller, H.R. Hilzinger, J. Magn. Magn. Mater. 2, 3 (1976).

[15] H. Kronmüller, K.-D. Durst, G. Martinek, J. Magn. Magn. Mater. 69, 149 (1987).

[16] D. Givord, A. Lienard, P. Tenaud, T. Viadieu, J. Magn. Magn. Mater. 67, L281 (1987). 
[17] D.W. Taylor, V. Villas-Boas, Q. Lu, M.F. Rossigol, F.P. Missel, D. Givord, S. Hirosawa, J. Magn. Magn. Mater. 130, 225 (1994).

[18] K. Kobayashi, D. Givord, J.M.D Coey, J. Magn. Magn. Mater. 157/158, 97 (1996).

[19] X.C. Kou, F.R. de Boer, R. Grössinger, G. Wiesinger, H. Suzuki, H. Kitazawa, T. Takamasu, G. Kido, J. Magn. Magn. Mater. 177-181, 1002 (1998).

[20] J.J. Becker, J. Appl. Phys. 39, 1270 (1968).

[21] D. Givord, P. Tenaud, T. Viadieu, J. Magn. Magn. Mater. 72, 247 (1988).

[22] J.J. Wysłocki, Phys. Status Solidi A 153, 487 (1996).

[23] E. Kondorsky, J. Phys. USSR 2, 161 (1940).

[24] N.P. Suponev, R.M. Grechishkin, M.B. Lyakhova, Yu, E. Pushkar, J. Magn. Magn. Mater. 157/158, 376 (1996).

[25] H. Kronmüller, T. Schrefl, J. Magn. Magn. Mater. 129, 66 (1994). 